\title{
CMEARTICLE
}

\section{Clinics in diagnostic imaging (144)}

Steven BS $\underline{\text { Wong }}^{1}$, MBChB, MMed, Theresa LC ${\underline{L e e^{2}}}^{2}$ BSc, MBBS, FRANZCR, Bruce B Forster ${ }^{3,4}, \mathrm{MSc}, \mathrm{MD}, \mathrm{FRCR}$, Gordon T Andrews ${ }^{4}$, MD, FRCPC

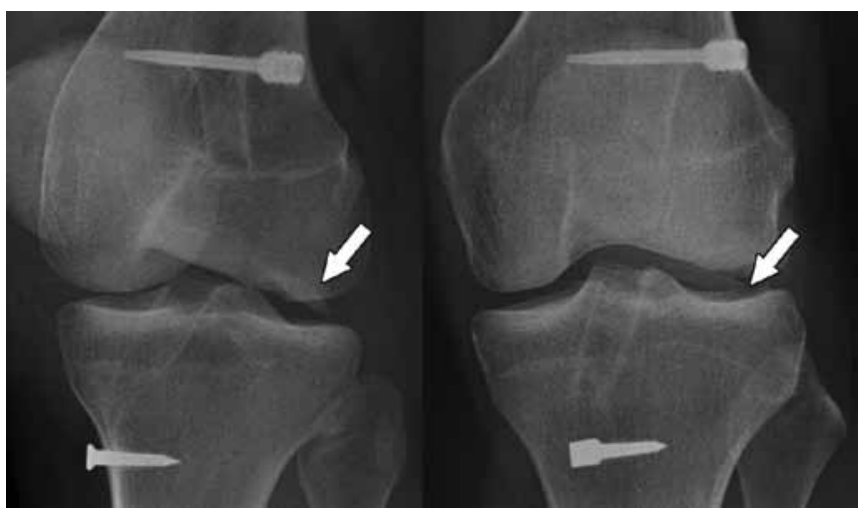

Fig. 1 Oblique and anteroposterior radiographs of the left knee joint show the distal femoral and proximal tibial tunnels for the anterior cruciate ligament neoligament.

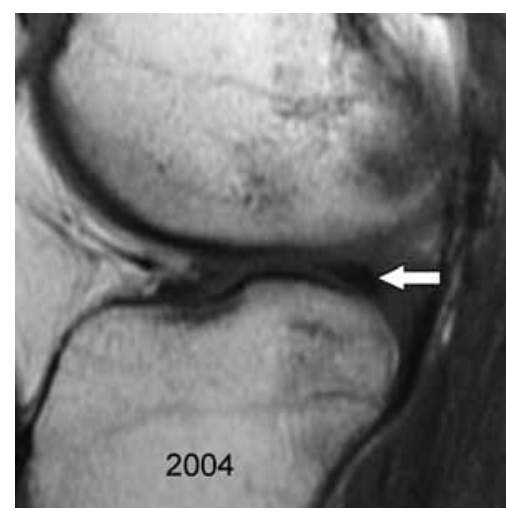

Fig. 3 Sagittal T1-W MR image of the left knee after the skiing incident in 2004 at the identical corresponding location show the scan parameters and positional differences.
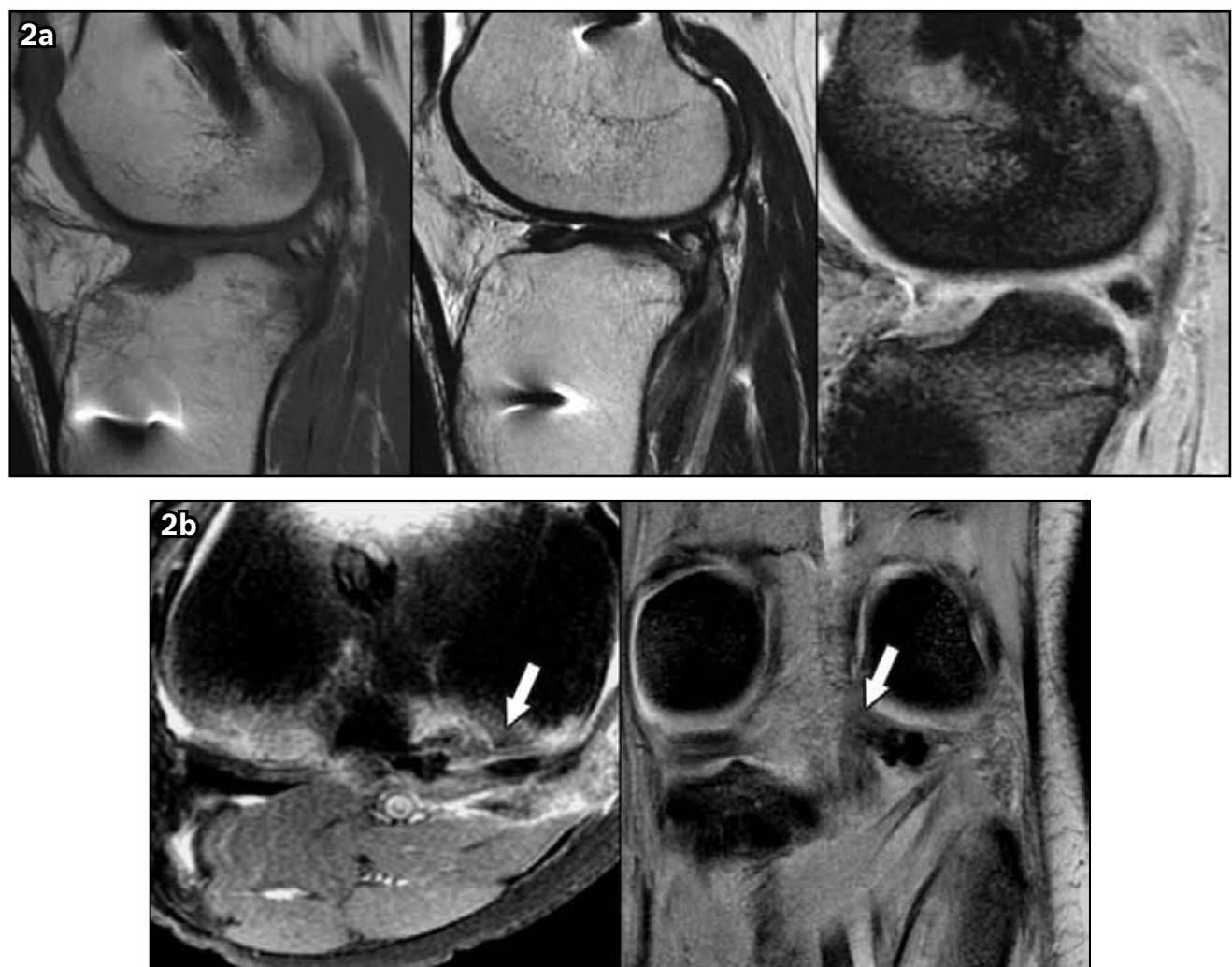

Fig. 2 (a) Sagittal T1-W, T2-W proton density (PD) and gradient echo (GRE) MR images of the left knee joint show the posterior aspect of the lateral femorotibial compartment. (b) Axial GRE and coronal T2-W PD images of the left knee joint show the posterior aspect of the lateral femorotibial compartment.

\section{CASE PRESENTATION}

A 35-year-old female patient who had an anterior cruciate ligament (ACL) repair done six years ago underwent magnetic resonance (MR) imaging of the left knee joint, after having sustained a recent traumatic patellar subluxation. The initial $\mathrm{ACL}$ tear had occurred during a skiing incident six years prior. At the current presentation, the patient was unable to fully extend her knee. Radiographs and MR images were obtained (Figs. 1 \& 2). What do these imaging studies show? How does it differ from the MR imaging study (Fig. 3) performed following the skiing injury?

${ }^{1}$ Department of Diagnostic Radiology, Singapore General Hospital, Singapore, ${ }^{2}$ PRP Diagnostic Imaging, Sydney, Australia, ${ }^{3}$ Department of Radiology, University of British Columbia, ${ }^{4}$ Diagnostic Radiology, University of British Columbia Hospital, Vancouver, Canada

Correspondence: Dr Steven Bak Siew Wong, Consultant Radiologist, Department of Diagnostic Radiology, Singapore General Hospital, Outram Road, Singapore 169608. drsbswong@hotmail.com 


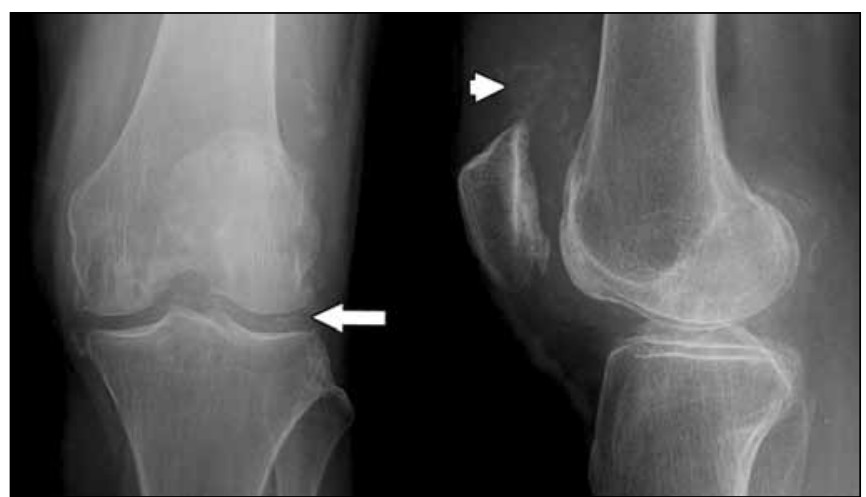

Fig. 4 Lateral and frontal radiographs show chondrocalcinosis in the lateral femorotibial joint space (arrow) and small irregular calcific opacities consistent with loose bodies in the suprapatellar space (arrowhead) in a knee with moderate severity osteoarthritis.

\section{IMAGE INTERPRETATION}

The radiographs (Fig. 1), obtained shortly after the recent patellar subluxation, demonstrate the presence of an intra-articular calcific structure (arrows in Fig. 1) projecting over the joint space of the lateral femorotibial compartment of the left knee joint. The distal femoral and proximal tibial tunnels, as well as their anchoring screws, are due to the previous ACL tendon graft reconstruction surgery. The current MR images of the left knee joint (Fig. 2) show the presence of an ossified structure within the posterior horn of the lateral meniscus (arrows in Fig. 2b), isointense to medullary bone in the distal femur and proximal tibia on the T1- and T2-weighted proton density sequences. On the sagittal and axial gradient echo sequences, the characteristic hypointense signal indicates the presence of calcium within the ossific structure. No intra-articular loose body was identified. Other imaging findings included a complete tear of the ACL neoligament, acute osteochondral compression fracture of the anterior weight-bearing aspect of the lateral femoral condyle, $\mathrm{ACL}$ injury pattern bone marrow oedema, disruption of the medial patellofemoral ligament and medial retinaculum, and lateral collateral ligament Grade 1 injury (not shown in figures). Of note, the ossified structure was not present on the MR image performed following the skiing injury six years ago (arrow in Fig. 3 denotes the corresponding area).

\section{DIAGNOSIS}

Lateral meniscal ossicle.

\section{CLINICAL COURSE}

The patient subsequently underwent arthroscopic partial local meniscectomy with successful removal of the meniscal ossicle. She made good recovery post-surgery.

\section{DISCUSSION}

With a reported prevalence of under $0.2 \%$, meniscal ossicles are relatively uncommon encounters in the everyday practice of most radiologists.(1) Although rare, they are reasonably wellknown entities, with sporadic reports published in Orthopaedic and Radiology literature. Meniscal ossicles have been reported in

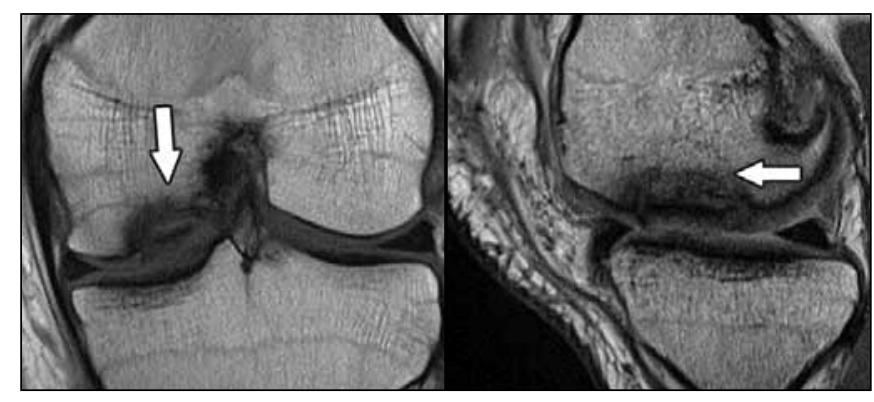

Fig. 5 Coronal and sagittal T1-W turbo spin-echo (TSE) MR sequences show osteochondritis dissecans with an undisplaced articular surface fragment (arrows) on the lateral weight-bearing aspect of the medial femoral condyle.
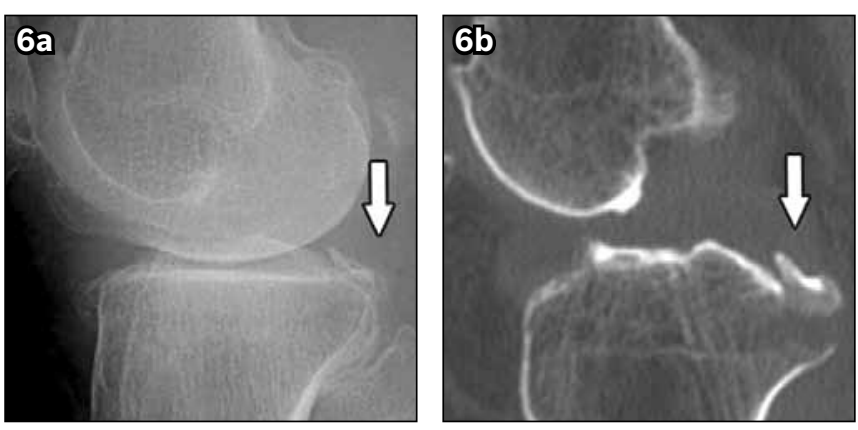

Fig. 6 (a) Lateral plain radiograph shows a posterior joint line bone fragment (arrow). (b) The corresponding nonenhanced sagittal reformatted CT image reveals this to be an avulsed fracture fragment (arrow) at the posterior cruciate ligament insertion site.

both male and female patients, with a higher incidence in young males. ${ }^{(2-4)}$ Most cases involve the posterior horn of the medial meniscus, with fewer reports of involvement in the lateral meniscus and anterior horns. ${ }^{(2,3,5)}$ Meniscal ossicles are defined as mature bone embedded within the substance of the meniscus. ${ }^{(5,6)}$ Histologically, ossicles are seen as lamellar and cancellous bone with fatty bone marrow, and they are surrounded by meniscal fibrocartilage. ${ }^{(1,6,7)}$ Several authors have reported foci of increased vascularisation, fibroblastic proliferation and even osteogenic activity. ${ }^{(2)}$

Patients either are asymptomatic or present with persistent or increasing knee pain, with reports of catching or clicking. In some patients, meniscal ossicles are incidental findings on radiographs or MR imaging. More often, they are discovered post referral for MR imaging after such calcific radio-opacity or 'loose bodies' have been reported on plain radiographs. The cancellous centre of the meniscal ossicle may not be evident on plain radiographs. It has been thought that the symptoms are related to the alteration of the contour of the meniscus. Associated meniscal tears have been reported and postulated as owing to alteration in the morphology of the meniscus. ${ }^{(5,6)}$

The main differential diagnosis remains an intra-articular loose body. Other differential diagnoses include chondrocalcinosis, osteochondritis dissecans or bony avulsion of several structures, such as the tibial attachment of the posterior cruciate ligament, the semi-membranosus or popliteus tendon or the meniscal posterior horns (meniscofemoral ligament). ${ }^{(2,7)}$ Synovial osteochondromatosis 

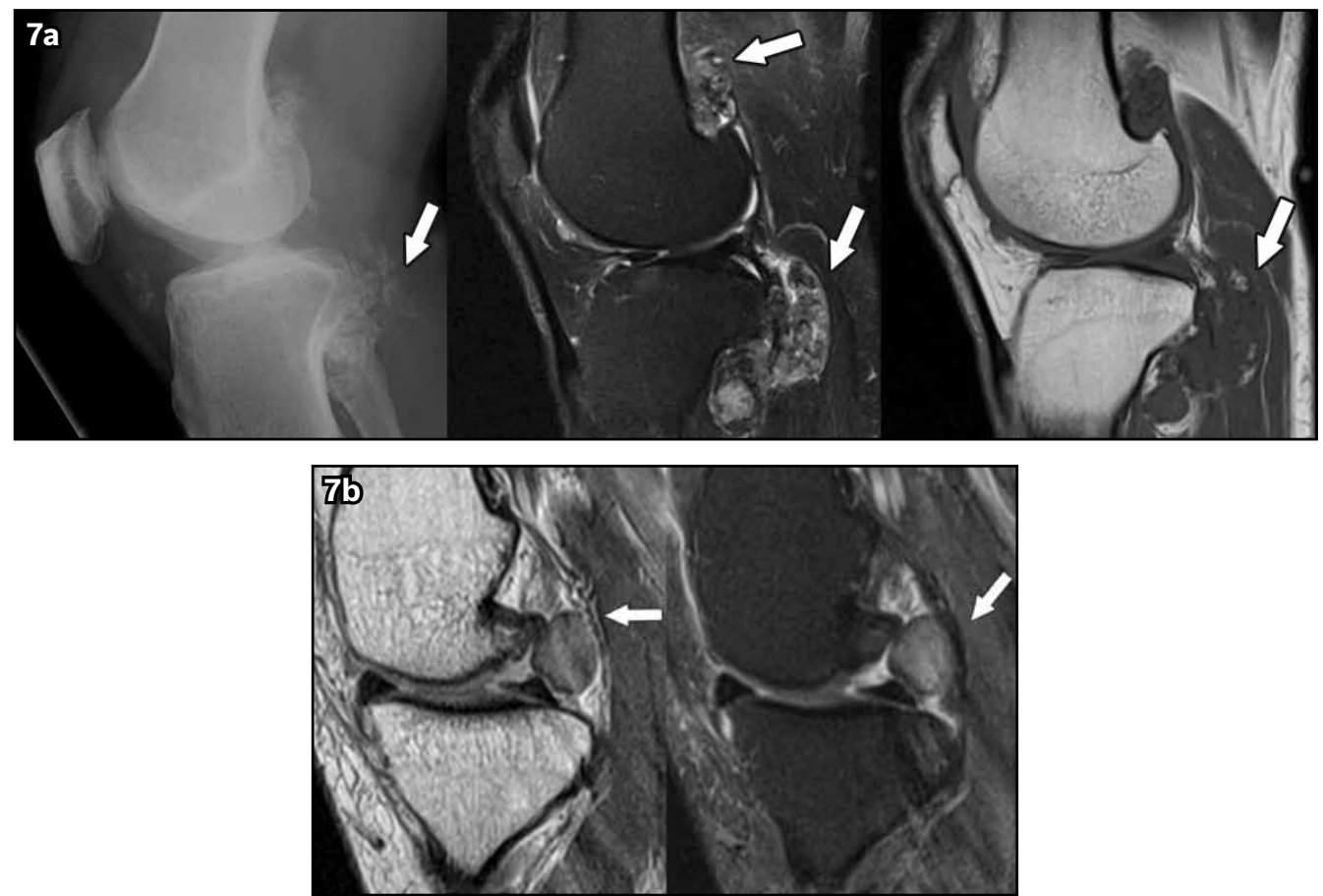

Fig. 7 (a) Lateral radiograph, and sagittal PD TSE and T1-W TSE MR images show multiple small millet-sized ossific bodies in the posterior knee joint compartment from synovial osteochondromatosis (arrows). On MR imaging, these are seen as T1-W hypointense to the bone marrow and T2-W isotense-to-hyperintense to the bone. (b) T1-W TSE and PD TSE MR images show a single, large mass (arrows) in the posterior joint space, abutting the posterior cruciate ligament. The T1-W hypointense and T2-W hyperintense signal characteristics are consistent with synovial osteochondromatosis.

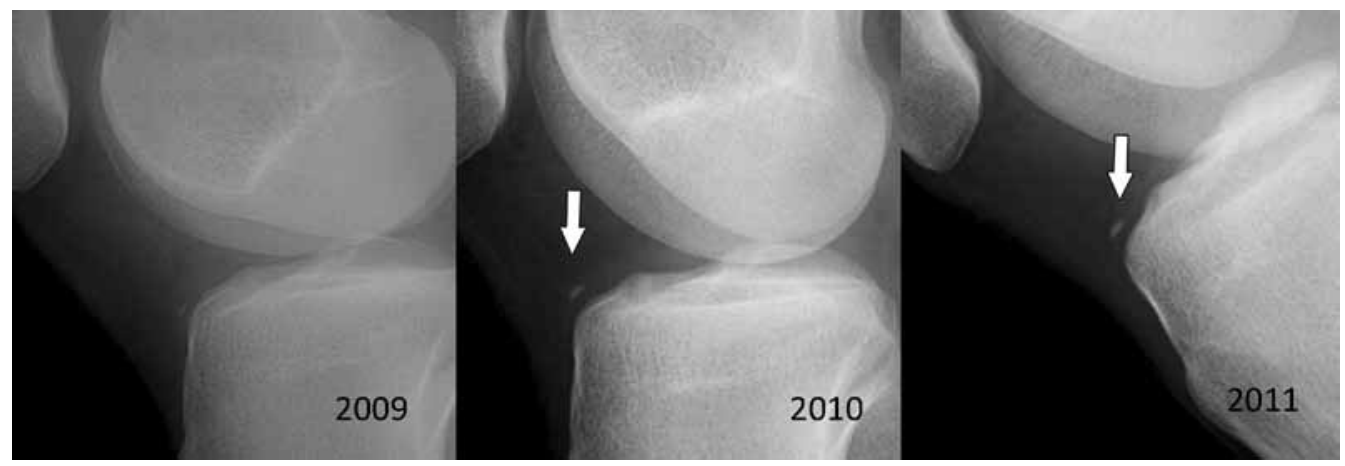

Fig. 8 Serial yearly radiographs of another patient show gradual ossification of a meniscal ossicle (arrows) within the anterior horn of the meniscus on lateral views.

is a less likely consideration given the multiplicity of the calcific bodies (Figs. 4-7).

The aetiology of meniscal ossicles remains uncertain, with several theories still being considered. One main theory postulates that they represent vestigial structures within the human knees, as meniscal ossicles are commonly seen in primates, cats and dogs. ${ }^{(6-9)}$ Another possibility is that they are post-traumatic sequelae, with metaplasia and heterotopic ossification within the meniscus occurring secondary to the trauma. ${ }^{(1,2,4)}$ Finally, it has been proposed that meniscal ossicles occur following avulsion of the posterior horn of the meniscus with the detachment of a fragment of the posterior tibial margin (Fig. 8). ${ }^{(4,10)}$

On imaging, meniscal ossicles may be seen on plain radiographs as intra-articular calcific corticated lesions, often related to the margins of the tibial plateau. A lucent centre may occasionally be discernible, indicating the presence of fatty bone marrow. Often, they are mistaken for intra-articular loose bodies. Fluoroscopic examination of the knee joints would demonstrate their relative immobility relative to the tibial plateau, although this is now rarely performed. ${ }^{(2)}$ Ultrasonography may show ossicles as hyperechoic structures with dense posterior acoustic shadowing, fixed within the meniscus, but visualisation with this modality can be difficult. ${ }^{(3)}$ Computed tomography (CT) imaging of the knee joint can confirm the presence of a calcific structure in the location of the meniscus, although absolute confirmation that they are embedded within the meniscus may not be possible. CT arthrography to demonstrate the location of the meniscal ossicle within the meniscus has been reported, but it is not often performed in this clinical scenario. ${ }^{(3)}$

MR imaging is the modality of choice for demonstrating meniscal ossicles, as it is capable of demonstrating the intrasubstance location within the meniscus, thus differentiating 

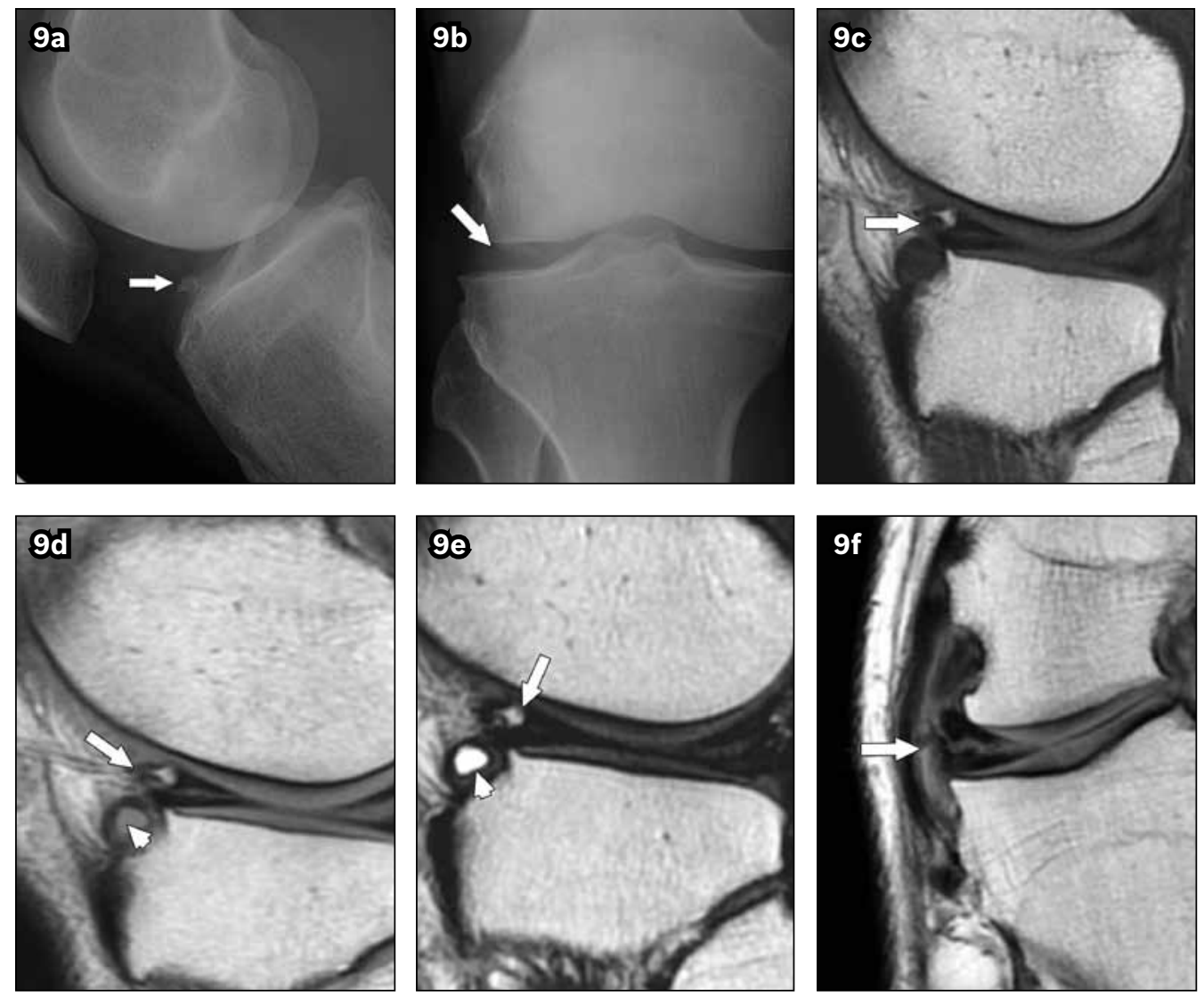

Fig. 9 Plain radiographs and MR images of another 38-year-old male patient with worsening knee pain following a twisting injury some years prior to presentation. (a) Lateral knee radiograph shows a small meniscal ossicle projecting over the anterior horn of the lateral meniscus of the right knee (arrow). (b) Frontal knee radiograph shows the meniscal ossicle, visible in the lateral femorotibial joint space (arrow). (c-e) The meniscal ossicle is demonstrated within the substance of the anterior horn of the lateral meniscus on (c) sagittal GRE sequence; (d) sagittal T1-W; and (e) sagittal PD MR images (arrows). Note the small parameniscal cyst anterior to the anterior horn, related to the full-thickness meniscal tear (arrowheads). (f) Coronal MR image shows a full-thickness horizontal tear of the body of the lateral meniscus in addition to the intrasubstance ossific structure (arrow).

the ossicle from an intra-articular loose body, and shows a typical signal pattern. Meniscal ossicles show MR imaging signals that are characteristic of normal bone with fatty marrow - centrally hyperintense on both T1- and T2-weighted images or fully corticated bone, and hypointense on most sequences, ${ }^{(2,3)}$ especially gradient echo sequences, due to susceptibility effects. Most ossicles are less than $10 \mathrm{~mm}$ in size. ${ }^{(4)}$ There may be thinning of the adjacent tibial articular cartilage, likely from pressure effect ${ }^{(6)}$ or associated meniscal tears. Joint effusion is rarely reported. Correlation with plain knee radiographs is helpful for confirming the presence of ossific structures.

Their intrameniscal location is the main feature that distinguishes meniscal ossicles from intra-articular loose bodies, chondrocalcinosis, bony avulsion of tendinous structures such as semimembranosus and popliteal tendons, and synovial osteochondromatosis. Loose bodies and chondrocalcinosis are generally more hypointense to the adjacent bone marrow on MR imaging than the more isointense meniscal ossicles. ${ }^{(7)}$ There is usually focal bone marrow oedema or bone defect at the site of the tendinous avulsion, whereas meniscal ossicles tend to indent the articular cartilage itself. In osteochondritis dissecans, articular cartilage defect is often associated with irregular margins. ${ }^{(4)}$ Synovial osteochondromatosis generally presents as multiple intra-articular bodies as compared to the solitary nature of the meniscal ossicle (Fig. 9).

Treatment for meniscal ossicles is usually arthroscopic resection, although some patients have opted for conservative management. ${ }^{(1,3,7)}$ It is beneficial for orthopaedic surgeons to have prior knowledge of the presence of a meniscal ossicle when planning an arthroscopic surgery, as this will prevent an unnecessary and fruitless search for a nonexistent intra-articular loose body.

\section{CONCLUSION}

Meniscal ossicles remain an uncommon finding on imaging of the knee joint. Although more often reported in the posterior horn of the medial meniscus, our patients had ossicles in uncommon locations - the anterior and posterior horns of their lateral meniscus. These cases support the post-traumatic theory of meniscal ossicle aetiology, as these patients all have a prior history of trauma. Recognition and differentiation of meniscal ossicles from intraarticular loose bodies is important, as this would have an impact on the surgical management of the patient. 
ABSTRACT A 35-year-old female patient with previous left knee anterior cruciate ligament repair for a skiing injury presented six years later with a traumatic lateral patellar subluxation. Radiographs and magnetic resonance imaging of her left knee joint showed an ossific structure in the region of the lateral meniscus. This was diagnosed as a meniscal ossicle and confirmed during successful arthroscopic excision. The imaging features of meniscal ossicles are reported.

Keywords: knee, meniscal ossicle, trauma

Singapore Med J 2013; 54(2):108-113

\section{REFERENCES}

1. Raustol OA, Poelstra KA, Chhabra A, Diduch DR. The meniscal ossicle revisited: etiology and an arthroscopic technique for treatment. Arthroscopy 2006; 22:687:e1-3.
2. Yu JS, Resnick D. Meniscal ossicle: MR imaging appearance in three patients. Skeletal Radiol 1994; 23:637-9.

3. Prabhudesai V, Richards PJ. Radiological appearance as a meniscal ossicle develops: a case report and review of literature. Injury 2003; 34:378-82.

4. Schnarkowski P, Tirman P, Fuchigami K, et al. Meniscal ossicle: Meniscal ossicle: radiographic and MR imaging findings. Radiology 1995; 196:47-50.

5. Yen P, Hunter J, Escobedo E. Meniscal ossicle in the lateral meniscus: an unusual variant of a rare entity. Radiology Case Reports [Online Journal] 2009; 4. Available at: http://radiology.casereports.net/index.php/rcr/article/ viewArticle/289/606. Accessed November 7, 2012.

6. Tuite MJ, De Smet AA, Swan JS, Keene JS. MR Imaging of a meniscal ossicle. Skeletal Radiol 1995; 24:543-5.

7. Rohilla S, Yadav RK, Singh R, Devgan A, Dhaulakhandi DB. Meniscal ossicle. J Orthop Traumatol 2009; 10:143-5.

8. Martinoli C, Bianchi S, Spadola L, Garcia J. Multimodality imaging assessment of meniscal ossicle. Skeletal Radiol 2000; 29:481-4.

9. Walker M, Phalan D, Jensen J, et al. Meniscal ossicles in large non-domestic cats. Vet Radiol Ultrasound 2002; 43:249-54.

10. Berg EE. The meniscal ossicle: the consequence of a meniscal avulsion. Arthroscopy 1991; 7:241-3. 


\section{SINGAPORE MEDICAL COUNCIL CATEGORY 3B CME PROGRAMME} (Code SMJ 201302B)

Question 1. Regarding meniscal ossicles:

(a) The incidence of reported cases is higher in young males.

(b) The lateral meniscus is more commonly involved than the medial meniscus.

(c) In some cases, meniscal ossicles are incidental findings on knee radiographs.

(d) They are entirely intrameniscal in location.

Question 2. The differential diagnoses of meniscal ossicles include:
(a) Torn displaced meniscus.
(b) Avulsion fractures related to the anterior cruciate ligament.
(c) Commonly misdiagnosed as synovial osteochondromatosis.
(d) Chondrocalcinosis is easily distinguishable from meniscal ossicles.

Question 3. The imaging features of meniscal ossicles are:
(a) They can occur anywhere within the knee joint.
(b) Lucent centres are common radiographic findings.
(c) Examination under fluoroscopic screening is commonly performed.
(d) CT arthrography is an accepted investigation option for evaluation.

Question 4. Regarding MR imaging of meniscal ossicles:

(a) Meniscal ossicles typically show fatty marrow signal centrally.

(b) Pressure effects on adjacent articular cartilage are always seen.

(c) Meniscal ossicles are isointense to adjacent bone marrow.

(d) Intravenous contrast medium administration is required to confirm the diagnosis on MR imaging.

True False

$\square \quad \square$

$\square$

$\square$

$\square$
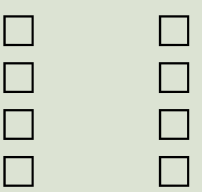

Question 5. Regarding the management of meniscal ossicles:

(a) Only meniscal ossicles larger than $10 \mathrm{~mm}$ in size require removal.

(b) Arthroscopic resection is the main treatment option.

(c) The management is different from that of an intra-articular loose body.

(d) Treatment requires total meniscectomy to prevent recurrence.

\footnotetext{
Doctor's particulars:

Name in full

MCR number

Email address

Specialty:

SUBMISSION INSTRUCTIONS:

(1) Log on at the SMJ website: http://www.sma.org.sg/publications/smjcurrentissue.aspx and select the appropriate set of questions. (2) Provide your name, email address and MCR number. (3) Select your answers and click "Submit".

RESULTS:

(1) Answers will be published in the SMJ April 2013 issue. (2) The MCR numbers of successful candidates will be posted online at the SMJ website by 1 April 2013. (3) Passing mark is $60 \%$. No mark will be deducted for incorrect answers. (4) The SMJ editorial office will submit the list of successful candidates to the Singapore Medical Council. (5) One CME point is awarded for successful candidates.

Deadline for submission: (February 2013 SMJ 3B CME programme): 12 noon, 25 March 2013.
} 